\title{
Optimization Methodology for Reconfigurable PV Modules
}

\author{
Patrizio Manganiello ${ }^{1,2}$, Pavlos Bosmalis ${ }^{1,3}$, Maro Baka ${ }^{4}$, Eszter Voroshazi ${ }^{1}$, Dimitrios Soudris ${ }^{4}$, Francky \\ Catthoor $^{1,2}$, Jozef Szlufcik ${ }^{1}$ and Jef Poortmans ${ }^{1,2,5}$ \\ ${ }^{1}$ imec, Kapeldreef 75, 3001 Heverlee, Belgium \\ 2 ESAT, KU Leuven, Kasteelpark Arenberg 10, 3001 Heverlee, Belgium \\ ${ }^{3}$ Aristotle University of Thessaloniki, University Campus, 54124 Thessaloniki, Greece \\ ${ }^{4}$ National Technical University of Athens, Heroon Polytechneiou 9, 15780 Athens, Greece \\ ${ }^{5}$ University of Hasselt, 3590 Diepenbeek, Belgium
}

\begin{abstract}
Reconfigurable photovoltaic modules represent an effective solution to improve $P V$ system resilience to partial shading. Indeed, the availability of different configurations increases energy generation under non-uniform conditions. However, the additional components that are active under uniform conditions lead to higher losses compared to equivalent static solutions. This paper presents a methodology for the design of optimized reconfigurable PV modules, balancing losses under uniform conditions and gain under partial shading. First, feasible reconfigurable module instantiations are selected given some design constraints. Then, the search space is further reduced by taking into account the typical operating conditions of the module. Finally, the best module layouts are chosen based on performance consideration. Results for a specific case study are presented to show the feasibility of the proposed methodology.
\end{abstract}

\section{INTRODUCTION}

One of the main drawbacks of conventional PV modules is their poor performance under non-uniform conditions, e.g. partial shading. Indeed, when part of a conventional PV module is shaded, either at least one entire subsection of the module is bypassed or the total module current is limited to the one of the worse performing cell. In both cases, most of the available energy is lost. Since applications such as Building-Integrated PV (BIPV), Building-Applied PV (BAPV) and solar vehicles are becoming widely employed in recent years, there is a clear need for PV module able to efficiently and effectively deal with the partial shading issue. Both static [1] and dynamically reconfigurable topologies [2]-[3] have been proposed. These latter require additional components, such as switches and submodule converters, to be able to perform the reconfiguration. By selecting at run-time the best configuration, reconfigurable modules generate much more energy than conventional modules under non-uniform conditions. However, the additional components that are active under uniform conditions lead to higher losses compared to equivalent static solutions. It is clear that a balance between losses under uniform conditions and gain under partial shading must be found.

Aim of this work is to present a methodology for the design of reconfigurable modules, able to explore and optimize this balance. The main focus in our current case study is the socalled Reconfigurable Snake PV (RSPV) modules [3].

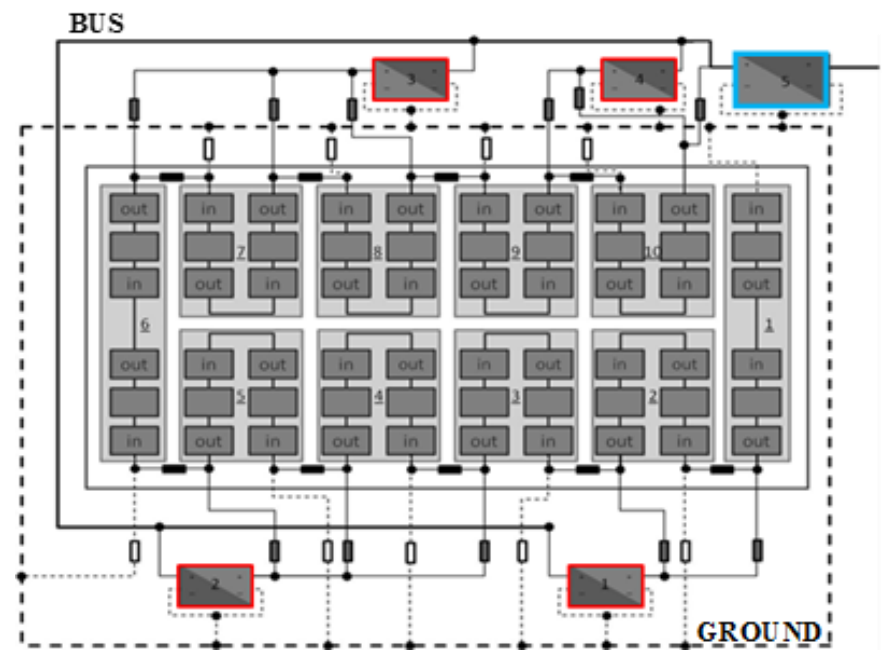

Fig. 1. I-type RSPV module.

However, the methodology can be easily extended to other types of reconfigurable PV modules.

\section{OPTIMIZATION METHODOLOGY}

Fig. 1 shows the "base" layout of a 60-cell I-type RSPV module. Switches between neighboring cell-strings (groups of cells statically connected), are used to connect all cell-strings in series under uniform conditions. When the module is subjected to non-uniform conditions, parallel connection of cell-strings is performed. Four local converters (red boxes) are employed to perform the Maximum Power Point Tracking (MPPT) and to reduce the overall current available on both the BUS line and the GROUND line when the cell-strings are connected in parallel. An additional converter (blue box) is used at the end of the module for connection to the rest of the PV array.

As shown in [4], the snake-like structure ensures minimal additional resistance due to the added wiring, as compared to the wiring scheme of a standard module, while keeping a reasonable level of granularity. Indeed, as further shown in [5], snake topologies made of 60 6-inch cells perform very well under partial shading, leading to $60-80 \%$ additional energy generation compared to standard topologies. However, their losses under uniform conditions may be unacceptably high [5]. 
Since uniform conditions are still expected to occur often, a reduction of these latter losses is critical. In order to achieve this, we need to explore the available parameters but a fully exhaustive enumeration of all alternatives would be prohibitive. So here we will propose a methodology to prune this huge space in a safe way so that we only keep the promising ones.

The additional loss of reconfigurable modules under uniform conditions mainly arises from the $\mathrm{ON}$ resistance of the reconfiguration switches that are used to connect cell-strings in series. A simplistic solution is the removal of some of these switches. However, this implies reduction of the total number of cell-strings $N_{C S}$ and it also makes some of them longer.

To still keep reasonable flexibility while reducing the number of cell-strings, the following design constraint is applied:

- cell-strings can only have two sizes. For instance, for the layout of Fig. 1, only cell-strings made of 6 cells (CS6) and 12 cells (CS12) must be considered

Other design constraints can be defined considering the parallel connection of cell-strings. More in details:

- Only neighboring cell-strings are connected to the same local converter, to keep the length of the wires between cell-strings and converter as small as possible

- Only cell-strings of the same size are connected in parallel, since they share a similar MPP voltage

- The maximum number of cell-strings connected in parallel to the same local converter is limited to 3 , to reduce converters' input current to reasonable values

- Number of local converters cannot be higher than 4, to keep the initial additional cost low

Such design constraints limit the number of possible groups of cell-strings that can be considered for parallel connection to a local converter. For instance, given the layout of Fig. 1, cellstrings can be grouped in only 6 different ways:

- $A=1 \times 6$, which is a group made of one CS6

- $B=1 \times 12$, which is a group made of one CS12

- $C=2 \times 6$, which is a group of two CS6 in parallel

- $D=2 \times 12$, which is a group of two CS12 in parallel

- $E=3 \times 6$, which is a group of three CS6 in parallel

- $F=3 \times 12$, which is a group of three CS12 in parallel

For a given number of local converters $N_{L C}$, still many different module instantiations can be obtained. However, promising instantiations must respect the following relation:

$$
\sum_{i=1}^{N_{L C}} k_{i} \cdot c_{i}=N
$$

Where $k_{i}=1,2,3$ is the number of cell-strings connected in parallel to the $i$-th local converter, $c_{i}=6,12$ represents the size of each cell-string connected in parallel to the $i$-th local converter and $N$ is the total number of cells in the module.

According to the groups of cell-strings defined above, any module instantiation may be described by a sequence of letters. For instance, $\{C E E C\}$ describes an instantiation in which 4 local converters are used and the cell-strings are grouped for parallel connection as following: (1) a group of two parallelconnected CS6 is connected to the first local converter; (2) a group of three parallel-connected CS6 is connected to the second local converter; (3) a group of three parallel-connected CS6 is connected to the third local converter; (4) a group of two parallel-connected CS6 is connected to the fourth local converter. Such instantiation is made in total of ten CS6, thus it verifies (1) if a 60 cells module is considered. $\{C E E C\}$ is indeed the descriptor for the module shown in Fig. 1. It is worth to note that $\{C E E C\}$ can be also seen as a multiset $M$ made of two unique elements, $C$ and $E$. Permutations of $\{C E E C\}$ represent different possible module instantiations.

Such representation can be used to further generalize the design process. Indeed, every triple $X=\left\{N_{C S}, N_{L C}, N\right\}$ can generate many multisets $M_{X, k}$ with number of unique elements equal to $C\left\{M_{X, k}\right\} \leq N_{L C}$. The multiplicity of the $n$-th unique element of the multiset $M_{X, k}$ is represented by $m_{n}$. Thus, every multiset $M_{X, k}$ allows for a total number of different configurations $N_{\text {conf }, M_{X, k}}$ equal to the multinomial coefficient

$$
\left(m_{1}, m_{2}, \ldots, m_{\mathrm{C}\left\{M_{X, k}\right\}}\right)=\frac{N_{L C} !}{m_{1} ! \cdot m_{2} ! \cdots \cdots m_{\mathrm{C}\left\{M_{X, k}\right\}} !}
$$

Every multiset that verifies (1) is representative of feasible module instantiations.

Let's show the process of selection of feasible module instantiation for the specific case $X=\{8,4,60\}$, that represents Snake modules made of 60 cells, with 8 cell-strings and 4 local converters. According to the design constraints described above, only three multisets verifying (1) can be created:

- $M_{X, 1}=\{B B E E\}$, where $m_{B}=m_{E}=2$

- $M_{X, 2}=\{A C D E\}$, where $m_{A}=m_{C}=m_{D}=m_{E}=1$

- $M_{X, 3}=\{C C C D\}$, where $m_{C}=3$ and $m_{D}=1$

Any other multiset doesn't verify (1), so it doesn't represent feasible module instantiations. The number of different instantiations for every multiset can be evaluated using (2), thus obtaining $N_{\text {conf }, M_{X, 1}}=6, N_{\text {conf }, M_{X, 2}}=24$ and $N_{\text {conf }, M_{X, 3}}=4$.

So, 36 different instantiations of such module $X$ are feasible.

\section{OPERATING SCENARIOS}

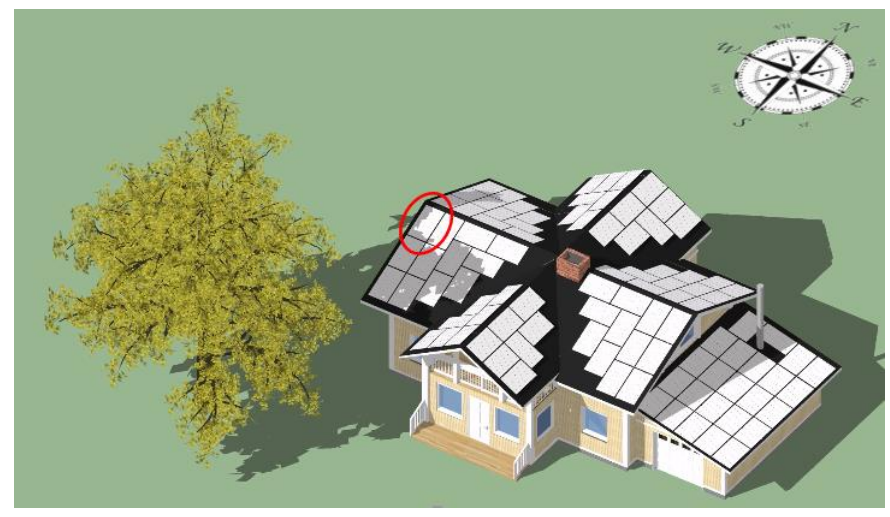

Fig. 2. Shading Scenario \#1. Shade is casted by a tree during part of the afternoon 
TABLE I

DAILY ENERGY GENERATION OF RSPV MODULES (WH)

\begin{tabular}{|c|c|c|c|c|c|c|c|c|c|c|c|c|c|}
\hline \multirow{3}{*}{$N_{C S}$} & \multirow{3}{*}{$N_{L C}$} & \multicolumn{4}{|c|}{ Scenario \#1 } & \multicolumn{4}{|c|}{ Scenario \#2 } & \multicolumn{4}{|c|}{ Scenario \#3 } \\
\hline & & \multicolumn{2}{|c|}{$\begin{array}{c}\boldsymbol{N}=\mathbf{6 0} \\
\text { Full Cells }\end{array}$} & \multicolumn{2}{|c|}{$\begin{array}{c}\boldsymbol{N}=\mathbf{1 2 0} \\
\text { Halved Cells }\end{array}$} & \multicolumn{2}{|c|}{$\begin{array}{c}\quad N=\mathbf{6 0} \\
\text { Full Cells }\end{array}$} & \multicolumn{2}{|c|}{$\begin{array}{c}\boldsymbol{N}=\mathbf{1 2 0} \\
\text { Halved Cells }\end{array}$} & \multicolumn{2}{|c|}{$\begin{array}{c}\quad N=\mathbf{6 0} \\
\text { Full Cells }\end{array}$} & \multicolumn{2}{|c|}{$\begin{array}{c}\boldsymbol{N}=\mathbf{1 2 0} \\
\text { Halved Cells }\end{array}$} \\
\hline & & I-Type & U-Type & I-Type & U-Type & I-Type & U-Type & I-Type & U-Type & I-Type & U-Type & I-Type & U-Type \\
\hline 10 & 4 & 1029 & 1050 & 1061 & 1074 & 1133 & 1168 & 1185 & 1192 & 1310 & 1312 & 1344 & 1348 \\
\hline \multirow{2}{*}{8} & 4 & 1048 & 1056 & 1057 & 1069 & 1161 & 1172 & 1183 & 1190 & 1315 & 1316 & 1341 & 1341 \\
\hline & 3 & 1039 & 1044 & 1046 & 1068 & 1159 & 1166 & 1175 & 1184 & 1307 & 1314 & 1338 & 1337 \\
\hline 6 & 3 & 1030 & 1036 & -- & -- & 1104 & 1157 & -- & -- & 1302 & 1299 & -- & -- \\
\hline 5 & 2 & N.A. & 1030 & N.A. & -- & N.A. & 1139 & N.A. & -- & N.A. & 1296 & N.A. & -- \\
\hline
\end{tabular}

Simulations have been performed using the framework presented in [5]. It allows for combination of measured weather data with simulated non-uniform and dynamic shading scenarios, so that realistic time- and spatially-varying operating scenarios are created. It also ensure high accuracy thanks to the use of a physics-based modeling approach. One of the simulated scenarios in shown in Fig. 2.

In the shading scenario shown in Fig. 2, shade is casted by a tree during part of the afternoon. Shade is highly irregular and often covers the entire module. In the following, we refer to such scenario as "Shading Scenario \#1". A slightly different building has been also considered in order to generate two additional shading scenarios where more regular shades are casted by the chimney during different parts of the day. In the following, we refer to such shading scenarios as "Shading Scenario \#2", shown in Fig. 3, and "Shading Scenario \#3", shown in Fig. 4. In all these cases, irradiance and ambient temperature data from Oldenburg (Germany) [6] are used.

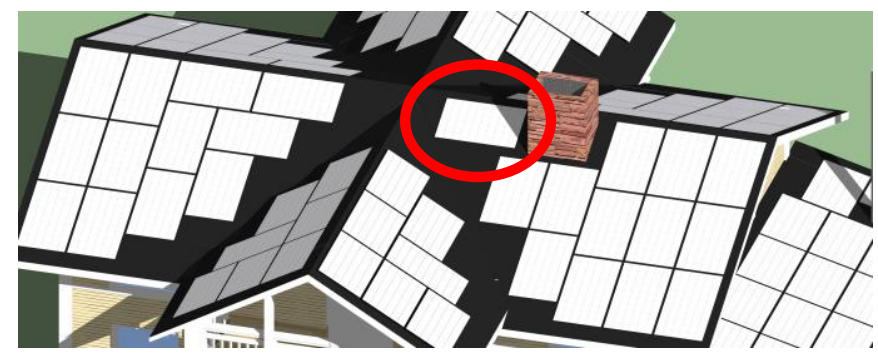

Fig. 3. Shading Scenario \#2. Shade is casted by the chimney during part of the morning.

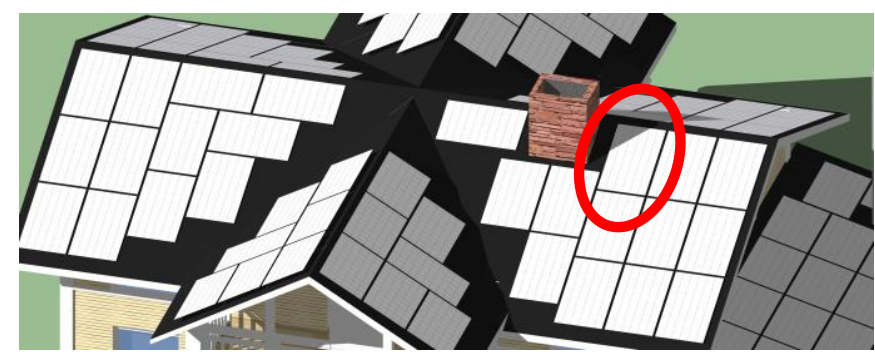

Fig. 4. Shading Scenario \#3. Shade is casted by the chimney during part of the afternoon
An additional step of the proposed optimization methodology arises here. Indeed, the number of instantiations to simulate using such framework can be further pruned based on the characteristics of the shading scenario. For instance, if a specific part of the module is mainly affected by partial shading during the day, it makes sense to position the shorter cell-strings in this part. This way, the module will show higher resilience to shading since the part of the module mainly subjected to shading will have higher granularity than the rest of the module.

\section{SIMULATION RESULTS}

RSPV modules made of (1) 60 6-inch full cells, organized in a 6x10 matrix as the one of Fig. 1, and (2) 120 6-inch halved cells, organized in a 12x10 matrix have been studied. Modules are modeled as described in [5]. The different instantiations to analyze have been selected according to the methodology described above.

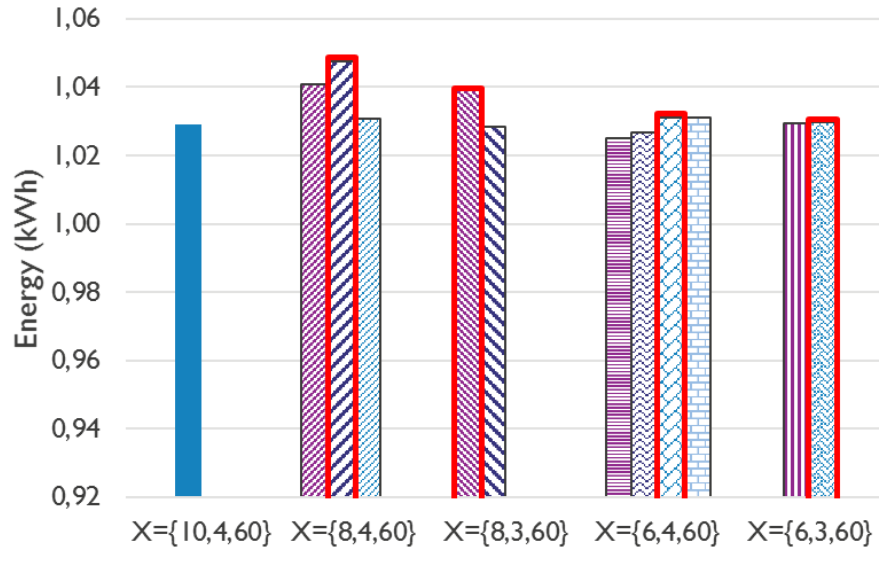

Fig. 5. Daily energy generation. I-Type full-cell RSPV, Shading Scenario \#1. Every bar represents a module instantiation, organized per group $X$.

Fig. 5 shows the simulation results for the I-Type full-cell RSPV module under Shading Scenario \#1. It is evident that higher energy can be extracted by reducing the number of cell- 
strings from 10 to 8 . This is mainly due to higher energy production under uniform conditions. Reducing the number of cell-strings down to 6 instead does not allow for higher energy production as power gains under uniform conditions are compensated by losses under shading. Since topologies belonging to the same group $X$ share similar costs (they have indeed the same number of local converters and the same number of reconfiguration switches), one module instantiation can be finally selected for each $X$. Namely, the ones that exhibit the best performance, as shown by the bars with red borders in Fig. 5.

Following the same approach for the other RSPV templates and the other shading scenarios, the simulation results summarized in Table I have been obtained. For the U-Type fullcell RSPV modules, the same conclusions of the I-Type fullcell RSPV modules can be drawn. Half-cell RSPV modules instead exhibit the absolute highest energy production when 10 cell-strings and 4 local converters are used. This is mainly due to the lower current produced by halved cells, that lead to a significant reduction of losses under both uniform and nonuniform conditions. It is worth to note that, for such RSPV template, reduction of the number of cell-strings never leads to higher energy production, since the lower losses under uniform conditions cannot compensate the lower energy generation under shading (due to reduced flexibility and granularity).

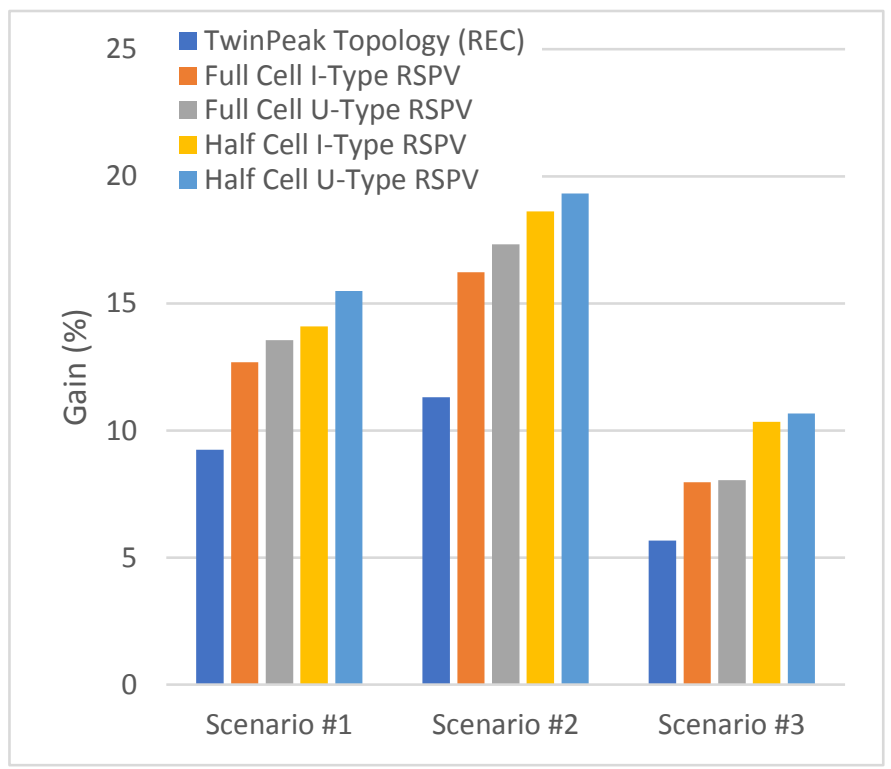

Fig. 6. Performance of different module topologies. Energy gains with respect to conventional module.

Optimized RSPV modules, i.e. RSPV modules allowing for the highest energy production according to the optimization procedure, have been benchmarked to current standard solutions as conventional PV modules (60 6-inch cells, 3 bypass diodes) and half-cell PV modules (120 6-inch halved cells, 3 bypass diodes) based on TwinPeak layout proposed by REC [1]. Fig. 6 shows the energy gain of the different module topologies with respect to conventional PV module under the three simulated shading scenarios.

Gain depends not only on the module topology, but also on the characteristics of the casted shade. Under Shading Scenario $\# 2$, unconventional topologies allow for the largest gains. This is due to the fact that, under Shading Scenario \#2: (1) partial shading is affecting a large part of the module; (2) such partial shading happens during morning hours, i.e. when ambient temperature is lower so that power generation is higher; (3) shade's daily evolution represents nearly worst-case for conventional modules, since shade often affects at least two substrings out of three. Shading Scenario \#3 instead leads to the smallest gains, since: (1) a smaller part of the module is shaded, namely only the top half of it; (2) partial shading is happening during afternoon, where cell power production is lowered due to higher ambient temperature; (3) shade slowly moves along the shorter edge of the module, meaning that the different substrings of the conventional module get slowly covered by shade during the afternoon, with only one substring partially shaded during peak hours. Finally, gains under Shading Scenario \#1 are in between the ones of the other two scenarios. Indeed, the large and highly irregular shade that is casted significantly reduces the power production of conventional modules but also affects in a non-negligible way the power generation of any other topology.

However, trends are maintained per topology along the different shading scenarios. Thanks to the use of halved cells as well as to a topology based on parallel connection of opposite substrings, TwinPeak topology performs better than conventional modules, since it gains energy under both uniform and partial shading conditions. The combination of these two gain mechanisms leads to an overall energy gain in the range $[5.6 \div 10.9] \%$ compared to conventional modules under the simulated shading scenarios.

RSPV modules outperform both conventional and TwinPeak topologies for the given shading scenarios, thanks to their higher granularity (8-10 substrings instead of 3-6) and to the use of local converters instead of bypass diodes. Best performance is shown by the U-Type half-cell RSPV module, that allows for up to $19 \%$ additional energy generation compared to a conventional module.

\section{CONCLUSION AND FUTURE WORK}

In this paper, a methodology for the optimization of reconfigurable PV modules has been presented. It allows to select feasible module instantiations given some design constraint and further reduce the search space by taking into account the typical operating conditions of the module. As a final results, the configurations leading to the best balance between losses under uniform conditions and gains under partial shading can be selected using the proposed methodology. Simulations have been performed under different realistic time- and spatial-varying operating conditions to 
demonstrate the feasibility of the proposed methodology and optimize both I-Type and U-Type RSPV modules made of 60 6-inch cells and 120 6-inch halved cells. However, the methodology can be easily extended for optimization of different reconfigurable module templates.

Moreover, both number of cell-strings and number of converters would be reduced, the additional initial cost of the module decreases. Relevant module selection methodology should include beyond performance also a financial analysis. Indeed, life-long energy gain must be monetized and compared to the initial additional cost.

\section{ACKNOWLEDGEMENT}

The authors gratefully acknowledge imec's SiPV industrial affiliation program and its partners. This project has received funding from the European Union's Horizon 2020 research and innovation programme under the Marie Skłodowska-Curie grant agreement No. 751159.

\section{REFERENCES}

[1] http://www.recgroup.com/sites/default/files/documents/whitepap er twinpeak shading properties eng.pdf

[2] G. Spagnuolo, et al., "Control of Photovoltaic Arrays: Dynamical Reconfiguration for Fighting Mismatched Conditions and Meeting Load Requests". IEEE Industrial Electronics Magazine, vol. 9, no. 1, pp. 62-76, March 2015.

[3] M. Baka, et al., "Smart PV Module Topology with a Snake-Like Configuration", 31st EU PVSEC, 2015, pp. 104-107.

[4] P. Bauwens, et al., "Reconfigurable Topologies for Smarter PV Modules: Simulation, Evaluation and Implementation", 32nd EU PVSEC, 2016, pp. 61-65

[5] P.Manganiello, et al.,"A bottom-up energy simulation framework to accurately compare PV module topologies under non-uniform and dynamic operating conditions", 44 ${ }^{\text {th }}$ IEEE PVSC, 2017

[6] http://www.unioldenburg.de/en/physics/research/ehf/energiemeteorology/meas urements/ 